Reprinted with permission of Kluwer Academic Publishers

J. S. Byrnes and J. F. Byrnes (eds.), Recent Advances in Fourier Analysis and its Applications, 441-454.

(C) 1990 Kluwer Academic Publishers.

\title{
GABOR AND WAVELET EXPANSIONS
}

\author{
Christopher Heil and David Walnut \\ The MITRE Corporation \\ 7525 Colshire Drive \\ McLean, Virginia 22102 \\ USA
}

\begin{abstract}
This paper is an examination of techniques for obtaining Fourier series-like expansions of finite-energy signals using so-called Gabor and wavelet expansions. These expansions decompose a given signal into time and frequency localized components. The theory of frames in Hilbert spaces is used as a criteria for determining when such expansions are good representations of the signals. Some results on the existence of Gabor and wavelet frames in the Hilbert space of all finite-energy signals are presented.
\end{abstract}

\section{Introduction.}

The frequency analysis of signals by expanding them in terms of a fixed collection of sinusoids, i.e., of representing the signal by means of its Fourier transform, has long been a useful technique in mathematics and engineering. However, the fact that the Fourier transform is not localized in time can make it an unnatural way of representing a signal. For example, music can be thought of as a signal in which the frequency content is changing over time since the combination of notes being received by the ear is constantly changing. One would like to define a transform which reflects this evolutionary nature of the spectrum of a signal. Gabor and wavelet transforms are one means of accomplishing this. They are generalizations of the ordinary Fourier transform defined for periodic functions in the sense that they give Fourier series-like expansions of signals which display both the time and frequency content of the signal.

Gabor-type expansions were introduced in the 1940s by D. Gabor [Gab]. He proposed the representation of an arbitrary signal as a sum of translated and modulated Gaussian functions. Gabor's idea can be illustrated by a decades-old technique known as the Short-time Fourier transform in which Fourier transforms are taken of short consectuive segments of a given signal. This transform gives an unambiguous representation of the signal and also gives a frequency picture of the 
signal locally in time.

To describe the Gabor transform, first fix a "windowing" function $g$. In order to analyze an arbitrary signal $f$, one forms the product of $f$ with a shifted version of $g$, i.e., $f(t) \overline{g(t-n a)}$ (where the bar indicates complex conjugation), then computes the Fourier series coefficients of this product, i.e.,

$$
c_{m n}(f)=\int_{\mathbf{R}} f(t) \overline{g(t-n a)} e^{-2 \pi i m b t} d t,
$$

for $m, n \in \mathbf{Z}$, the set of integers. If $g$ is concentrated at 0 , then the coefficient $c_{m n}(f)$ should to some extent give the intensity of the frequency $m b$ at time $n a$. It is hoped that, as $n$ and $m$ range over all integers, the coefficients $c_{m n}(f)$ completely determine $f$ and that some sense can be made of the "Fourier series" representation

$$
f(t) \sim \sum_{m, n} c_{m n}(f) g(t-n a) e^{2 \pi i m b t}
$$

The accuracy of the above representation is highly dependent on the function $g$ and the values of the parameters $a$ and $b$.

The other type of expansion examined here is known as wavelet (or sometimes affine wavelet) expansion. The transform associated with this type of expansion was introduced by A. Grossman and J. Morlet as a way of analyzing seismic signals [GGM]. There has been a great deal of recent work done using wavelets in this and related areas. For example, they have been used in the analysis of images [Mal], sound patterns $[\mathrm{KMG}]$, and in numerical matrix computation [BCR]. As with the Gabor transforms, the point here is to represent a signal in a way which displays both the time and frequency content of the signal. The way this is accomplished in the wavelet case is as follows. Take a fixed function $\varphi$ (known as the mother wavelet) which is concentrated at 0 and consider the function $a^{n / 2} \varphi\left(a^{n} t\right)$. If $a>1$ and $n>0$ then this function is compressed in time and consequently expanded in frequency. We form the coefficients $c_{m n}(f)$ by integrating $f$ against translated versions of this function, i.e.,

$$
c_{m n}(f)=a^{n / 2} \int_{\mathbf{R}} f(t) \overline{\varphi\left(a^{n} t-m b\right)} d t
$$

For large $n>0$, the coefficient $c_{m n}(f)$ gives an idea of the high-frequency content of $f$ in a small time neighborhood about $a^{-n} m b$. As before, we want to make sense of the representation

$$
f(t) \sim \sum_{m, n} c_{m n}(f) a^{n / 2} \varphi\left(a^{n} t-m b\right) .
$$

The criterion we use to make sense of these representations comes from the theory of frames in Hilbert spaces. In this we follow the paper [DGM], where this 
connection was first made. The general concept of frames was first introduced by R. J. Duffin and A. C. Schaeffer in connection with non-harmonic Fourier series [DS]. The Hilbert space under consideration here will be $L^{2}(\mathbf{R})$, the space of all finite-energy signals on the real line $\mathbf{R}$. All of the results presented here can be generalized to more than one dimension. A theory in two dimensions is important for image analysis in particular.

The focus of this paper is on presenting results on the existence of Gabor frames and wavelet frames for the Hilbert space $L^{2}(\mathbf{R})$. The results here are due to others and a more detailed and complete treatment can be found in the expository paper $[\mathrm{HW}$. The first part is an introduction to Hilbert spaces and frames in Hilbert spaces. In Section 2, the Zak transform is introduced and results on the existence of Gabor frames for arbitrary $g \in L^{2}(\mathbf{R})$ and parameters $a, b$ such that $a b=1$ are given. In this case, it turns out that necessary and sufficient conditions can be given having to do with the Zak transform of $g$. In Section 3, we examine what can be done for finer lattices, that is, when $a b<1$. We present two existence theorems giving simple conditions on $g$ and $a$ which guarantee that we have a frame for all sufficiently small $b>0$. Section 4 deals with wavelet frames and we show by means of an example the sense in which the wavelet transform can be thought of as a time and frequency localization operator. This example leads to an existence theorem which appears in $[\mathrm{DGM}]$.

\section{Frames in Hilbert Spaces.}

In this section we will describe some of the basic properties of frames in Hilbert spaces, showing that they are useful generalizations of orthonormal bases. By a Hilbert space, we mean a vector space, $H$, which possesses an inner product $\langle x, y\rangle$ and which is complete in the norm $\|x\|=\langle x, x\rangle^{1 / 2}$ (for the precise meaning of these terms we refer the reader to $[\mathrm{GG}]$ ).

The only Hilbert space we will actually use in this paper is $L^{2}(\mathbf{R})$, the space of all complex-valued signals $f$ defined on the real line, $\mathbf{R}$, which have finite energy, i.e., for which

$$
\|f\|=\left(\int_{\mathbf{R}}|f(t)|^{2} d t\right)^{1 / 2}<\infty .
$$

The inner product in this Hilbert space is

$$
\langle f, g\rangle=\int_{\mathbf{R}} f(t) \overline{g(t)} d t
$$

where the bar indicates complex conjugation.

Frames were first introduced in 1952 by R. J. Duffin and A. C. Schaeffer in the paper [DS], in connection with nonharmonic Fourier series. Their first use in connection with wavelets was in 1986 in the paper [DGM] by I. Daubechies, A. Grossmann, and Y. Meyer. The precise definition is as follows. 
Definition 1.1. A frame for a Hilbert space $H$ is a set of vectors $\left\{x_{n}\right\}$ for which there exist constants $A, B>0$ such that

$$
A\|x\|^{2} \leq \sum_{n}\left|\left\langle x, x_{n}\right\rangle\right|^{2} \leq B\|x\|^{2}
$$

for every $x \in H$.

It is well-known that given any Hilbert space $H$, there always exists an orthonormal basis, i.e., a set of vectors $\left\{e_{n}\right\}$ such that

(1) $\left\langle e_{m}, e_{n}\right\rangle= \begin{cases}1, & \text { if } m=n \\ 0, & \text { if } m \neq n\end{cases}$

(2) $\sum\left|\left\langle x, e_{n}\right\rangle\right|^{2}=\|x\|^{2}$ for all $x \in H$.

Every orthonormal basis is clearly a frame with $A=B=1$. However, frames are much more general than orthonormal bases, for we do not place on them the stringent requirement of orthonormality (condition (1)), and we relax the equality in condition (2) to an inequality. A fundamental property of orthonormal bases is that every element $x \in H$ can be written in terms of the orthonormal basis in a unique way as $x=\sum\left\langle x, e_{n}\right\rangle e_{n}$. We shall see that frames also give representations of elements of the Hilbert space, although these representations need not be unique. However, they are still computable and under good control.

As a trivial example of a frame which is not an orthonormal basis, consider the following. Let $\left\{e_{1}, e_{2}, \ldots\right\}$ be an orthonormal basis for a Hilbert space $H$. This is surely then a frame with bounds $A=B=1$. However, the set

$$
\left\{e_{1} / \sqrt{2}, e_{1} / \sqrt{2}, e_{2} / \sqrt{2}, e_{2} / \sqrt{2}, \ldots\right\}
$$

is also a frame with bounds $A=B=1$, but is not an orthonormal basis.

In this paper we will be interested in constructing two specific types of frames for the Hilbert space $L^{2}(\mathbf{R})$. In each case, the frame elements have a particularly simple form, for they are functions which are generated from a single fixed function (called the mother wavelet) by combinations of the basic operations of translation, modulation, and dilation, defined by:

$\begin{array}{lll}\text { Translation: } & T_{a} f(x)=f(x-a), & \text { for } a \in \mathbf{R} ; \\ \text { Modulation: } & E_{a} f(x)=e^{2 \pi i a x} f(x), & \text { for } a \in \mathbf{R} ; \\ \text { Dilation: } & D_{a} f(x)=a^{-1 / 2} f(x / a), & \text { for } a>0 .\end{array}$

In Sections 2 and 3 we will construct frames of the form $\left\{g_{m n}\right\}_{m, n \in \mathbf{Z}}$, where

$$
g_{m n}(t)=e^{2 \pi i m b t} g(t-n a)=E_{m b} T_{n a} g(t)
$$

for some fixed function $g \in L^{2}(\mathbf{R})$ and fixed parameters $a, b>0$. Such frames will be called Weyl-Heisenberg, or $\mathbf{W}-\mathbf{H}$, frames, and we say that $g$ generates the 
frame. In Section 4 we discuss affine frames, which have the form $\left\{\varphi_{m n}\right\}_{m, n \in \mathbf{Z}}$, where

$$
\varphi_{m n}(t)=a^{n / 2} \varphi\left(a^{n} t-m b\right)=D_{a^{n}} T_{m b} \varphi(t)
$$

for some fixed $\varphi \in L^{2}(\mathbf{R})$ and fixed $a>1, b>0$. We will discuss conditions on $g$ and $\varphi$ under which we can be sure that Weyl-Heisenberg or affine frames will exist. First, however, we list in the remainder of this section some properties of general frames in Hilbert spaces. Most of these general results first appeared in [DS]; expanded proofs and statements also appear in [HW].

Theorem 1.2. If $\left\{x_{n}\right\}$ is a frame then the following hold.

(1) $S x=\sum\left\langle x, x_{n}\right\rangle x_{n}$ converges for all $x \in H$.

(2) $S$ is an isomorphism of $H$ onto itself, i.e., it is bijective and continuous, and has a continuous inverse.

(3) $x=\sum\left\langle x, S^{-1} x_{n}\right\rangle x_{n}$ for all $x \in H$, but not necessarily uniquely.

(4) If $x=\sum c_{n} x_{n}$ for some scalars $\left\{c_{n}\right\}$ then

$$
\sum_{n}\left|c_{n}\right|^{2}=\sum_{n}\left|\left\langle x, S^{-1} x_{n}\right\rangle\right|^{2}+\sum_{n}\left|c_{n}-\left\langle x, S^{-1} x_{n}\right\rangle\right|^{2}
$$

Condition (4) says that, while the representation $x=\sum\left\langle x, S^{-1} x_{n}\right\rangle x_{n}$ may not be unique, it is in fact the "best" way to write $x$ in terms of the $\left\{x_{n}\right\}$.

The following theorem gives a necessary and sufficient condition for uniqueness in frame representations.

Theorem 1.3. Given a frame $\left\{x_{n}\right\}$ in a Hilbert space $H$. Then the representations $x=\sum\left\langle x, S^{-1} x_{n}\right\rangle x_{n}$ are unique for every $x \in H$ if and only if there exists an orthonormal basis $\left\{e_{n}\right\}$ and an isomorphism $U$ of $H$ such that $x_{n}=U e_{n}$ for all $n$.

\section{Weyl-Heisenberg frames with lattice size 1.}

In this section we consider the problem of determining when an arbitrary $g \in L^{2}(\mathbf{R})$ will generate a $\mathrm{W}-\mathrm{H}$ frame if $a, b>0$ are such that $a b=1$. Recall that a $\mathrm{W}-\mathrm{H}$ frame has the form $\left\{g_{m n}\right\}_{m, n \in \mathbf{Z}}$, where $g_{m n}(t)=e^{2 \pi i m b t} g(t-n a)$. If one considers the lattice of points $\{(m b, n a)\}_{m, n \in \mathbf{Z}}$ in the plane, then the value $a b$ is the area of the rectangles in the plane determined by this lattice. It can be shown that the value $a b=1$ is a "critical value" for $\mathrm{W}-\mathrm{H}$ frames in that it is impossible to construct a $\mathrm{W}-\mathrm{H}$ frame if $a b>1$. We will see in this section that it is possible to construct $\mathrm{W}-\mathrm{H}$ frames when $a b=1$, and, moreover, such $\mathrm{W}-\mathrm{H}$ frames have the desirable feature that the frame representations are unique. Unfortunately, we will also find that only "bad" functions $g$ can generate W-H frames for this critical value, bad in the sense of either not being smooth or not having good decay. 
A crucial tool in this analysis is what has come to be called the Zak transform. This transform has been introduced independently by many groups in many different areas of pure and applied mathematics; in fact, a discrete version was used by Gauss [S]. J. Zak investigated it for quantum mechanical reasons beginning in the 1960s [Z]; recent work includes that of A. J. E. M. Janssen [J1; J2]. Proofs of the theorems in this section can be found in [J1] and [HW].

Definition 2.1. The Zak transform of a function $f \in L^{2}(\mathbf{R})$ is (formally)

$$
Z f(t, \omega)=a^{1 / 2} \sum_{k=-\infty}^{\infty} f(t a+k a) e^{2 \pi i k \omega}
$$

for $t, \omega \in \mathbf{R}$, and where $a>0$ is a fixed parameter.

The following facts about $Z$ are easily proved.

\section{Theorem 2.2.}

(1) $Z f$ is quasiperiodic, i.e.,

$$
\begin{aligned}
& Z f(t+1, \omega)=e^{-2 \pi i \omega} Z f(t, \omega) \\
& Z f(t, \omega+1)=Z f(t, \omega)
\end{aligned}
$$

Thus $Z f$ is completely determined by its values on the unit rectangle $Q=$ $[0,1] \times[0,1]$.

(2) The series defining $Z f$ converges in an $L^{2}$-sense on $Q . Z$ is an unitary map of $L^{2}(\mathbf{R})$ onto $L^{2}(Q)$, i.e., it is a norm-preserving isomorphism.

(3) $Z g_{m n}=E_{m n} \cdot Z g$, where

$$
E_{m n}(t, \omega)=e^{2 \pi i m t} e^{2 \pi i n \omega} .
$$

Now, since $Z$ is unitary, $\left\{g_{m n}\right\}$ will form a frame for $L^{2}(\mathbf{R})$ if and only if $\left\{Z g_{m n}\right\}$ forms a frame for $L^{2}(Q)$. But from Theorem $2.2, Z g_{m n}=E_{m n} \cdot Z g$, which, combined with the fact that $\left\{E_{m n}\right\}$ is an orthonormal basis for $L^{2}(Q)$, places great restrictions on the function $Z g$. In particular, we can prove the following theorem.

Theorem 2.3. Given $g \in L^{2}(\mathbf{R})$ and $a, b>0$ with $a b=1$. Then $\left\{g_{m n}\right\}$ forms $a$ frame if and only if there exist constants $A, B$ such that

$$
0<A \leq|Z g(t, \omega)|^{2} \leq B<\infty
$$

for almost every $(t, \omega) \in Q$.

This theorem implies that the frame representations provided by $\left\{g_{m n}\right\}$ will be unique. For, if $\left\{g_{m n}\right\}$ is a frame then $Z g$ will be essentially constant by Theorem 2.3, so the mapping $U$ defined on $L^{2}(Q)$ by $U F=F \cdot Z g$ is an isomorphism of $L^{2}(Q)$. Since $U E_{m n}=E_{m n} \cdot Z g=Z g_{m n}$ and $\left\{E_{m n}\right\}$ is an orthonormal basis, this implies by Theorem 1.3 that the frame representations are unique. This, of course, is a desirable feature. Unfortunately, we can show that only "bad" functions can have Zak transforms which are essentially constant in the sense of Theorem 2.3. 
Theorem 2.4. If $Z g$ is continuous, then it has a zero.

Proof. Assume $Z g$ was continuous but nonvanishing. Then we can find (see $[\mathrm{RR}]$ ) a continuous function $\varphi(t, \omega)$ such that

$$
Z g(t, \omega)=|Z g(t, \omega)| e^{i \varphi(t, \omega)}
$$

for $(t, \omega) \in Q$. Using the quasiperiodicity of $Z g$, it is not difficult to show that there must then exist integers $k, l$ such that

$$
\begin{aligned}
& \varphi(t+1, \omega)=\varphi(t, \omega)-2 \pi \omega+2 \pi k \\
& \varphi(t, \omega+1)=\varphi(t, \omega)+2 \pi l
\end{aligned}
$$

for all $(t, \omega) \in Q$. Hence,

$$
\begin{aligned}
0= & \varphi(0,0)-\varphi(1,0) \\
& +\varphi(1,0)-\varphi(1,1) \\
& +\varphi(1,1)-\varphi(0,1) \\
& +\varphi(0,1)-\varphi(0,0) \\
= & -2 \pi,
\end{aligned}
$$

a contradiction.

It is shown in [H1] that if $g$ is continuous and satisfies the mild decay condition

$$
\sum_{k=-\infty}^{\infty} \operatorname{ess} \sup _{t \in[k, k+1]}|g(t)|<\infty,
$$

then $Z g$ must be continuous, and therefore $g$ cannot generate a $\mathrm{W}-\mathrm{H}$ frame. Thus, for example, no smooth function with compact support, in fact, no Schwartz function, can generate a W-H frame at the critical value $a b=1$. In particular, the Gaussian function $g(t)=e^{-\pi t^{2}}$ will not generate a frame. A similar criteria is the following.

Theorem 2.5. If $g \in L^{2}(\mathbf{R})$ and

$$
\left(\int_{\mathbf{R}}|t g(t)|^{2} d t\right)\left(\int_{\mathbf{R}}|\gamma \hat{g}(\gamma)|^{2} d \gamma\right)<\infty,
$$

(where $\hat{g}$ is the Fourier transform of $g$ ) then $g$ cannot generate a $W$ - $H$ frame when $a b=1$.

See [Bal], [Bat], [L], [D3], [DJ], [BHW] for discussions and proofs of this theorem.

\section{Existence of $\mathbf{W}-\mathbf{H}$ frames for smaller lattices.}

As mentioned in Section 2, the value $a b=1$ is a critical value for $\mathrm{W}-\mathrm{H}$ frames, in that there are no $\mathrm{W}-\mathrm{H}$ frames when $a b>1$. Moreover, we showed that $\mathrm{W}-\mathrm{H}$ frames 
for $a b=1$ all have unique representations, but unfortunately can only exist when $g$ is either not smooth or has bad decay. In this section, we examine the effect of taking a smaller lattice size, i.e., of considering $a b<1$. It can be shown that the frame representations in this case will not be unique, but on the other hand we will see that very good functions $g$ will generate $\mathrm{W}-\mathrm{H}$ frames if we allow $a b$ to be small. Our goal is to prove existence theorems of the following form: given $g \in L^{2}(\mathbf{R})$ (the mother wavelet) and $a>0$, find conditions under which there is an interval $\left(0, b_{0}\right)$ such that $\left\{g_{m n}\right\}$ is a W-H frame for $L^{2}(\mathbf{R})$ for every $b \in\left(0, b_{0}\right)$.

The following theorem, which is a straightforward generalization of a theorem of Daubechies ([D3]), is a very general existence theorem and will be examined carefully in subsequent pages.

Theorem 3.1. Let $g \in L^{2}(\mathbf{R})$ and $a>0$ satisfy:

(3.1.1) there exist constants $A, B>0$ such that for almost every $x \in \mathbf{R}$ we have

$$
A \leq \sum_{n}|g(x-n a)|^{2} \leq B
$$

(3.1.2) $\lim _{b \rightarrow 0} \sum_{j \neq 0} \beta_{a}(j / b)=0$, where

$$
\beta_{a}(s)=\operatorname{ess} \sup _{x \in \mathbf{R}} \sum_{n}|g(x-n a) g(x-s-n a)| .
$$

Then there exists $b_{0}>0$ such that $\left\{g_{m n}\right\}$ is a frame for $L^{2}(\mathbf{R})$ for all $b \in\left(0, b_{0}\right)$.

A proof of this theorem can be found in [D3] and [HW]. Condition (3.1.1) is necessary in order that $\left\{g_{m n}\right\}$ form a frame. This condition can be thought of as an "overlapping" condition on the mother wavelet $g$. The existence of the lower bound $A$ means that the successive shifts of the function $|g|^{2}$ cover the entire real line and leave no "gaps". If there were such a gap, then any function supported in the gap would not be recognized by any of the wavelets $g_{m n}$. That is, we could find a non-zero function $f$ such that $\left\langle f, g_{m n}\right\rangle=0$ for all $m$ and $n$, which would imply that $\left\{g_{m n}\right\}$ was not a frame.

The existence of the bound $B$ says that $g$ must at least be bounded in order for it to generate a frame. The bound $B$ is required to have good control over the size of the wavelet coefficients over all the functions $f \in L^{2}(\mathbf{R})$.

If $g$ vanishes outside an interval $I$ of length at most $1 / b$ then $\beta_{a}(s)=0$ if $|s| \geq 1 / b$. In this case, the form of the frame operator is particularly simple, in fact,

$$
S f=\sum_{m, n}\left\langle f, g_{m n}\right\rangle g_{m n}(x)=f(x) \cdot b^{-1} \sum_{n}|g(x-n a)|^{2} .
$$

Thus, $S$ is an isomorphism if and only if $\sum|g(x-n a)|^{2}$ is bounded above and below, i.e., if and only if (3.1.1) is satisfied. In this case, then, (3.1.1) is both necessary and sufficient in order that $\left\{g_{m n}\right\}$ form a frame.

The condition (3.1.2) can be replaced by the simpler condition of the following theorem. The proof can be found in $[\mathrm{HW}]$. 
Theorem 3.2. Let $g \in L^{2}(\mathbf{R})$ and $a>0$ satisfy condition (3.1.1) and also

$$
\sum_{n=-\infty}^{\infty} \operatorname{ess}_{x \in[n, n+1)}|g(x)|<\infty .
$$

Then there exists $b_{0}>0$ such that $\left\{g_{m n}\right\}$ is a frame for $L^{2}(\mathbf{R})$ for all $b \in\left(0, b_{0}\right)$.

Condition (3.2.1) means that the sequence of maximum values of the function $|g|$ on successive intervals is summable. There are many examples of such functions, such as the Gaussian $g(x)=e^{-\pi x^{2}}$, and more generally any function $g$ for which there exists a $C>0$ and an $\epsilon>0$ such that

$$
|g(x)| \leq C(1+|x|)^{-(1+\epsilon)}
$$

for all $x \in \mathbf{R}$.

Theorem 3.2 is proved by showing that a function which satisfies condition (3.2.1) must also satisfy condition (3.1.2). This makes Theorem 3.2 less general than Theorem 3.1. However, that (3.2.1) implies (3.1.2) shows that condition (3.1.2) is a condition governed by the growth of the function $g$ at $\infty$. Also, (3.2.1) is a much easier condition to verify than (3.1.2).

\section{Affine Systems.}

In this section, we give a brief introduction to some aspects of wavelet decompositions of functions in $L^{2}(\mathbf{R})$. As in Sections 2 and 3, we will address the question of the existence of such decompositions and give an indication of why such decompositions might be useful in signal processing or image analysis by giving a physical interpretation to the expansion coefficients. As usual, the theory of frames in Hilbert spaces will be used as a criterion to determine whether arbitrary signals can be written down in terms of such a collection of functions.

Definition 4.1. Let $\varphi \in L^{2}(\mathbf{R})$ and $a>1, b>0$ be given. Then the (affine) wavelet system generated by $\varphi, a$, and $b$ is the set of functions $\left\{\varphi_{m n}\right\}_{m, n \in \mathbf{Z}}$, where

$$
\varphi_{m n}=a^{n / 2} \varphi\left(a^{n} t-m b\right) .
$$

We always assume that the function $\varphi$, the mother wavelet satisfies the condition

$$
\int_{\mathbf{R}} \varphi(t) d t=0
$$

This means that $\varphi$ (if it is real valued) has as much area above the axis as below, which gives the function a resemblance to a "wave". This is why the term "wavelet" was coined. 
The functions making up an affine system all arise from a single function under the action of a collection of norm-preserving transformations, namely, dilations and translations. Recall that the dilation operator $D_{\alpha}$ is defined by

$$
D_{\alpha} f(t)=\alpha^{1 / 2} f(\alpha t) \text {. }
$$

The factor $\alpha^{1 / 2}$ means that $D_{\alpha}$ preserves the $L^{2}$-norm of the functions it acts upon, that is,

$$
\int_{\mathbf{R}}\left|D_{\alpha} f(t)\right|^{2} d t=\int_{\mathbf{R}}|f(t)|^{2} d t
$$

If $\alpha>1$ then $D_{\alpha}$ has the effect of concentrating a function at the origin by making it narrower and (in order to perserve $L^{2}$-norm) taller. If $\alpha<1$ then $D_{\alpha}$ spreads the function out and decreases the amplitude. If we use the notation $T_{\beta}$ to denote translation by $\beta$ (as defined in Section 1), then we can write our affine system as

$$
\varphi_{m n}=D_{a^{n}} T_{m b} \varphi
$$

In order to give an interpretation of the physical meaning of the set of wavelet coefficients of a particular signal $f$, let us consider, for a fixed $n>0$, the collection of coefficients

$$
c_{m n}(f)=\left\langle f, \varphi_{m n}\right\rangle=a^{n / 2} \int f(t) \overline{\varphi\left(a^{n} t-m b\right)} d t .
$$

The coefficients $c_{m n}(f)$ represent comparisons of $f$ with successive shifts by $a^{-n} m b$ of the function $a^{n / 2} \varphi\left(a^{n} t\right)$. Since $n>0$ and $a>1$ we have $a^{n}>1$ and consequently this dilated function is highly concentrated at 0 . Intuitively, then, it should be the case that these comparisons pick out the high frequency behavior or fine detail of the signal $f$ in a small time interval around $a^{-n} m b$. This type of transform is often referred to as a scaling transform because the coefficients $c_{m n}(f)$ where $n$ is fixed, pick out those features of $f$ which exist on a time-scale of about $a^{-n} b$, so that as $n$ becomes large, the coefficients for that $n$ pick out smaller and smaller scale features of the signal. Features on a scale much smaller than $a^{-n} b$ are not noticed because they are averaged out when the coefficients are computed. Features on a scale much larger than $a^{-n} b$ are not noticed because of the shape of the mother wavelet $\varphi$, i.e., because $\int \varphi=0$. That is, if $f$ were nearly constant in a neighborhood of $a^{-n} m b$, then the value of the corresponding coefficient $c_{m n}(f)$ should be nearly zero. In language suggestive of image processing, these scales are often referred to as levels of resolution, and the wavelet transform as a multiresolution transform. Thus it is accurate to say that the coefficients $c_{m n}(f)$ essentially pick out only the high frequency behavior present in the signal $f$ at the given resolution level which was not present at the previous resolution level. For an image, one would say that these coefficients contain only the additional detail in the signal $f$ at this level of resolution which was not detectable at the previous level of resolution. 
We shall give a more precise mathematical formulation of the above rather vague statements by looking at the following simple example. Let $\varphi$ be a squareintegrable function (let us for convenience assume integrability as well) such that $\hat{\varphi}$ vanishes outside an interval of the form $[\ell, L]$, where $\ell, L>0$, and in fact is non-zero everywhere inside the interval. Since $\hat{\varphi}$ is continuous, this means that $\hat{\varphi}$ is bounded and that it is bounded below on subintervals of $[\ell, L]$. Let $b>0$ be such that $b^{-1}=L-\ell$ and $a>1$ such that $a<L / \ell$. Let us consider the collection $\left\{\varphi_{m n}\right\}$ for this $\varphi$ and the sum

$$
\sum_{m, n}\left\langle f, \varphi_{m n}\right\rangle \varphi_{m n}
$$

Notice that under the action of the Fourier transform, dilations on the time side go into dilations in the opposite sense on the frequency side, that is,

$$
\left(D_{\alpha} \varphi\right)^{\wedge}(\gamma)=D_{\alpha^{-1}} \hat{\varphi}(\gamma)
$$

Using this fact, the fact that translations on the time side go into modulations on the frequency side, and Parseval's formula, we can prove the following identity (for details, see $[\mathrm{HW}])$ :

$$
\left(\sum_{m}\left\langle f, \varphi_{m n}\right\rangle \varphi_{m n}\right)^{\wedge}(\gamma)=\hat{f}(\gamma) \cdot b^{-1}\left|\hat{\varphi}\left(a^{-n} \gamma\right)\right|^{2}
$$

Now, since $\hat{\varphi}$ vanishes outside the interval $[\ell, L]$, the function $\left|\hat{\varphi}\left(a^{-n} \gamma\right)\right|^{2}$ is supported in $\left[a^{n} \ell, a^{n} L\right]$. Thus the sum

$$
\sum_{m}\left\langle f, \varphi_{m n}\right\rangle \varphi_{m n}
$$

represents a band-filtered version of $f$ where the band is $\left[a^{n} \ell, a^{n} L\right]$. If $n>0$ then these contain the high frequencies of $f$ and if $n<0$, the low frequencies of $f$.

It should be noted here that since $\hat{\varphi}$ is supported on the right half-line in the frequency domain, only positive frequencies of $f$ will be measured by the wavelet coefficients and hence such a collection of wavelets can never be a frame for $L^{2}(\mathbf{R})$. There are several ways of handling this situation. If one is dealing with only realvalued signals $f$ (which is usually the case in practice) then it is enough to know $\hat{f}$ on the right (or left) half-line in order to completely determine $f$. Another possibility is to consider an additional function $\tilde{\varphi}$ which has the same properties as $\varphi$ only transferred to the left half-line in the frequency domain. For this purpose, it is enough to take $\tilde{\varphi}(t)=\varphi(-t)$. Finally, it is possible to find a function $\varphi$ whose Fourier transform is supported on both halves of the frequency axis such that the $\left\{\varphi_{m n}\right\}$ are a frame for $L^{2}(\mathbf{R})$. We shall see that the wavelet orthonormal basis of Y. Meyer is of this type. For details on such frame constructions see [HW]. 
The above example also gives some insight into the notion of the frame operator associated to a collection of wavelets as a "deblurring" operator. For fixed $M$ and $\varphi$ as before, arguing as above gives

$$
\left(\sum_{n \leq M} \sum_{m}\left\langle f, \varphi_{m n}\right\rangle \varphi_{m n}\right)^{\wedge}(\gamma)=\hat{f}(\gamma) \cdot b^{-1} \sum_{n \leq M}\left|\hat{\varphi}\left(a^{-n} \gamma\right)\right|^{2} .
$$

The function $\sum_{n \leq M}\left|\hat{\varphi}\left(a^{-n} \gamma\right)\right|^{2}$ is supported in $\left[0, a^{M} L\right]$ and in fact does not vanish on this interval. Thus one can see that the above sum is a low-pass filtered or "blurred" version of $f$. The effect of increasing $M$ to infinity is to add more and more of the high frequency characteristics (one might say "finer and finer details") of $f$ into the sum until finally a complete reconstruction is achieved.

We are now able to state and prove an existence theorem for affine frames for $L^{2}(\mathbf{R})$. This theorem appears in $[\mathrm{DGM}]$.

Theorem 4.2. Let $\varphi \in L^{2}(\mathbf{R})$ and $a>1, b>0$ satisfy

(4.2.1) $\hat{\varphi}$ is supported in $[\ell, L]$ where $L>\ell>0, b^{-1} \leq L-\ell$ and $1<a \leq l / \ell$,

(4.2.2) there exist constants $A, B>0$ such that for almost every $\gamma \in \hat{\mathbf{R}}$ we have

$$
A \leq \sum_{n}\left|\hat{\varphi}\left(a^{n} \gamma\right)\right|^{2} \leq B
$$

Then $\left\{\varphi_{m n}, \tilde{\varphi}_{m n}\right\}$ is a frame for $L^{2}(\mathbf{R})$, where $\tilde{\varphi}(t)=\varphi(-t)$.

Proof. The same type of Fourier series argument as before gives

$$
\sum_{m, n}\left|\left\langle f, \varphi_{m n}\right\rangle\right|^{2}=\int_{0}^{\infty}|\hat{f}(\gamma)|^{2} b^{-1} \sum_{n}\left|\hat{\varphi}\left(a^{n} \gamma\right)\right|^{2} d \gamma
$$

Therefore,

$$
\begin{aligned}
& \sum_{m, n}\left|\left\langle f, \varphi_{m n}\right\rangle\right|^{2}+\sum_{m, n}\left|\left\langle f, \tilde{\varphi}_{m n}\right\rangle\right|^{2} \\
& \quad=\int_{0}^{\infty}|\hat{f}(\gamma)|^{2} b^{-1} \sum_{n}\left|\hat{\varphi}\left(a^{n} \gamma\right)\right|^{2} d \gamma+\int_{-\infty}^{0}|\hat{f}(\gamma)|^{2} b^{-1} \sum_{n}\left|\hat{\tilde{\varphi}}\left(a^{n} \gamma\right)\right|^{2} d \gamma .
\end{aligned}
$$

Hence,

$$
\begin{aligned}
A \int_{-\infty}^{\infty}|f(t)|^{2} d t & =A \int_{-\infty}^{\infty}|\hat{f}(\gamma)|^{2} d \gamma \\
& \leq \sum_{m, n}\left|\left\langle f, \varphi_{m n}\right\rangle\right|^{2}+\sum_{m, n}\left|\left\langle f, \tilde{\varphi}_{m n}\right\rangle\right|^{2} \\
& \leq B \int_{-\infty}^{\infty}|\hat{f}(\gamma)|^{2} d \gamma \\
& =B \int_{-\infty}^{\infty}|f(t)|^{2} d t
\end{aligned}
$$


from which the result follows.

Finally, we state some results on the existence of affine systems which are actually orthonormal bases for $L^{2}(\mathbf{R})$. The differences in the theorems come from the smoothness and decay properties that the mother wavelets can have. In each case we take $a=2$ and $b=1$.

Theorem 4.3. The Haar system $\left\{D_{2^{n}} T_{m} \varphi\right\}$ is an orthonormal basis of compactly supported wavelets for $L^{2}(\mathbf{R})$. The Haar system is defined by taking $a=2, b=1$, and

$$
\varphi(t)= \begin{cases}1, & \text { if } 0 \leq t<1 / 2, \\ -1, & \text { if } 1 / 2 \leq t<1 \\ 0, & \text { otherwise. }\end{cases}
$$

Theorem 4.4 (Meyer). There exists a function $\varphi \in L^{2}(\mathbf{R})$ such that

(1) $\hat{\varphi}$ is infinitely differentiable and the support of $\hat{\varphi}$ is contained in the compact set $[-4 / 3,-1 / 3] \cup[1 / 3,4 / 3]$ so that in particular, $\varphi$ is a Schwartz function, that is, it and all of its derivatives are continuous and decay faster than any polynomial.

(2) $\int \varphi(t) t^{k} d t=0$ for every integer $k$.

(3) $\left\{D_{2^{n}} T_{m} \varphi\right\}$ is an orthonormal basis for $L^{2}(\mathbf{R})$.

Theorem 4.5 (Lemarie). Given $N>0$, there is a function $\varphi \in L^{2}(\mathbf{R})$ such that

(1) $\varphi$ has $N$ continuous derivatives, and

$$
|\varphi(t)| \leq C e^{-\alpha t}
$$

for some $C, \alpha>0$.

(2) $\int \varphi(t) t^{k} d t=0$ for $k=0,1,2, \ldots, N+1$.

(3) $\left\{D_{2^{n}} T_{m} \varphi\right\}$ is an orthonormal basis for $L^{2}(\mathbf{R})$.

Theorem 4.6 (Daubechies). Given a number $N>0$, there is a function $\varphi \in$ $L^{2}(\mathbf{R})$ such that

(1) $\varphi$ has $N$ continuous derivatives, and $\varphi$ has compact support.

(2) $\int \varphi(t) t^{k} d t=0$ for $k=0,1,2, \ldots, N-1$.

(3) $\left\{D_{2^{n}} T_{m} \varphi\right\}$ is an orthonormal basis for $L^{2}(\mathbf{R})$.

\section{REFERENCES}

[Bal] R. BALIAN, Un principe d'incertitude fort en théorie du signal ou en mécanique quantique, C. R. Acad. Sci. Paris 292 (1981), 1357-1362.

[Bat] G. Battle, Heisenberg proof of the Balian-Low theorem, Lett. Math. Phys. 15 (1988), $175-177$.

[Ben] J. Benedetto, Gabor representations and wavelets, Commutative Harmonic Analysis, 1987, D. Colella, ed., Contemp. Math. 91, American Mathematical Society, Providence, 1989, 9-27. 
[BHW] J. Benedetto, C. Heil, And D. Walnut, Remarks on the proof of the Balian theorem, preprint.

[BCR] G. Beylkin, R. Coifman, And V. Rokhlin, Fast wavelet transforms and numerical algorithms, I, preprint.

[D1] I. DAubechies, Orthonormal bases of compactly supported wavelets, Comm. Pure Appl. Math. 41 (1988), 909-996.

[D2] - Time-frequency localization operators: a geometric phase space approach, IEEE Trans. Inform. Theory 34 (1988), 605-612.

[D3] - The wavelet transform, time-frequency localization and signal analysis, IEEE Trans. Inform. Theory, to appear.

[DGM] I. Daubechies, A. Grossmann, And Y. Meyer, Painless nonorthogonal expansions, J. Math. Phys. 27 (1986), 1271-1283.

[DJ] I. Daubechies And A. J. E. M. JAnssen, Two theorems on lattice expansions, IEEE Trans. Inform. Theory, to appear.

[DS] R. J. Duffin And A. C. Schaeffer, A class of nonharmonic Fourier series, Trans. Amer. Math. Soc. 72 (1952), 341-366.

[Gab] D. Gabor, Theory of communications, J. Inst. Elec. Eng. (London) 93 (1946), 429-457.

[GG] I. Gohberg And S. GoldBerg, Basic Operator Theory, Birkhäuser, Boston, 1981.

[Gro] A. Grossmann, Wavelet transforms and edge detection, Stochastic Processing in Physics and Engineering, S. Albeverio et al., eds., D. Reidel, Dordrecht, the Netherlands, 1988, pp. $149-157$.

[GGM] P. Goupillaud, A. Grossmann, And J. Morlet, Cycle-octave and related transforms in seismic signal analysis, Geoexploration 23 (1984/85), 85-102.

[GM] A. Grossmann And J. Morlet, Decomposition of Hardy functions into square integrable wavelets of constant shape, SIAM J. Math. Anal. 15 (1984), 723-736.

[H1] C. HEIL, Wiener amalgam spaces in generalized harmonic analysis and wavelet theory, Ph.D. thesis, University of Maryland, College Park, MD, 1990.

[H2] Wavelets and frames, Proceedings of the Summer Signal Processing Conference, Institute for Mathematics and Its Applications, Minneapolis, MN, August, 1988.

[HW] C. Heil And D. WAlnut, Continuous and discrete wavelet transforms, SIAM Review 31 (1989), 628-666.

[J1] A. J. E. M. JANSSEN, Bargmann transform, Zak transform, and coherent states, J. Math. Phys. 23 (1982), 720-731.

[J2] - The Zak transform: a signal transform for sampled time-continuous signals, Philips J. Res. 43 (1988), 23-69.

[KMG] R. Kronland-Martinet, J. Morlet, and A. Grossmann, Analysis of sound patterns through wavelet transforms, Internat. J. Pattern Recog. Artif. Int. 1 (1987), 273-302.

[LM] P. LemariÉ And Y. Meyer, Ondelettes et bases hilbertiennes, Rev. Mat. Iberoamericana 2 (1986), 1-18.

[L] F. Low, Complete sets of wave packets, A Passion for Physics-Essays in Honor of Geoffrey Chew, C. DeTar, et al., ed., World Scientific, Singapore, 1985, pp. 17-22.

[Mal] S. MALlat, A theory for multiresolution signal decomposition: the wavelet representation, IEEE Trans. on Pattern Anal. and Machine Intel. 11 (1989), 674-693.

[Mey] Y. MeYer, Principe d'incertitude, bases hilbertiennes et algèbres d'opérateurs, Séminaire Bourbaki 662 (1985-86).

[RR] T. RAdo And P. Reichelderfer, Continuous Transformations in Analysis, SpringerVerlag, Berlin, New York, 1955.

[S] W. SchempP, Radar ambiguity functions, the Heisenberg group, and holomorphic theta series, Proc. Amer. Math. Soc. 92 (1984), 103-110.

[W] D. WALnut, Weyl-Heisenberg wavelet expansions: existence and stability in weighted spaces, Ph.D. thesis, University of Maryland, College Park, MD, 1989. 
[Y] R. Young, An Introduction to Nonharmonic Fourier Series, Academic Press, New York, 1980.

[Z] J. ZAK, Finite translations in solid state physics, Phys. Rev. Lett. 19 (1967), 1385-1397. 